Case Report

\title{
Sticky Platelet Syndrome: An Unrecognized Cause of Acute Thrombosis and Graft Loss
}

\author{
Fabio Solis-Jimenez $\mathbb{D}^{1},{ }^{1}$ Hector Hinojosa-Heredia $\mathbb{D}^{0},{ }^{2}$ Luis García-Covarrubias, ${ }^{2}$ \\ Virgilia Soto-Abraham, ${ }^{3}$ and Rafael Valdez-Ortiz $\mathbb{D}^{4}$
}

${ }^{1}$ Internal Medicine Service, General Hospital of Mexico "Dr. Eduardo Liceaga”, Mexico City, Mexico

${ }^{2}$ Transplant Service, General Hospital of Mexico "Dr. Eduardo Liceaga”, Mexico City, Mexico

${ }^{3}$ Pathological Anatomy Service, General Hospital of Mexico "Dr. Eduardo Liceaga", Mexico City, Mexico

${ }^{4}$ Nephrology Service, General Hospital of Mexico "Dr. Eduardo Liceaga”, Mexico City, Mexico

Correspondence should be addressed to Fabio Solis-Jimenez; fabiosolisjimenez@gmail.com and Rafael Valdez-Ortiz; rafavaldez@gmail.com

Received 28 December 2017; Accepted 13 March 2018; Published 22 April 2018

Academic Editor: Sophia Lionaki

Copyright (C) 2018 Fabio Solis-Jimenez et al. This is an open access article distributed under the Creative Commons Attribution License, which permits unrestricted use, distribution, and reproduction in any medium, provided the original work is properly cited.

Introduction. Sticky platelet syndrome (SPS) is a prothrombotic disease that is not well recognized and difficult to diagnose. Case Report. We present a case of a 49-year-old diabetic woman on ambulatory peritoneal dialysis therapy who underwent a kidney transplant from living-related donor. The donor was her sister with whom she shared one haplotype and absence of donor specific antibodies. The posttransplant evolution was torpid, developing progressive deterioration, which made us suspect a failure in the graft. Doppler ultrasound reported renal vein thrombosis and hypoperfusion of the renal artery. Without clinical improvement, she required a reintervention that ended in graftectomy, in which the histopathological report showed negative C4d with medullary and cortical infarction. Hematological studies were negative for antibodies against phospholipids, with correct levels of proteins $\mathrm{C}$ and $\mathrm{S}$ and antithrombin. Platelet aggregometry studies were carried out, which were compatible with SPS. Conclusions. Recognition of SPS in pretransplant studies is difficult if there is no history of previous thrombotic events. However, we must consider this entity in cases of acute thrombosis and loss of the graft of uncertain origin.

\section{Introduction}

Primary graft thrombosis occurs in $0.5 \%$ and $6 \%$ of renal transplants and usually results in graft loss [1]. Risk factors associated with the development of graft thrombosis have been identified, such as the use of peritoneal dialysis, retransplantation, prolonged cold ischemia (greater than 24 hours), and transsurgical hypotension [2-4]. However, in all patients presenting with thrombosis of the graft, an intentional search for possible primary thrombophilias should be carried out [4]. Retrospective studies performed in transplant patients with graft thrombosis have shown a higher incidence of protein $\mathrm{C}$, protein $\mathrm{S}$, factor $\mathrm{V}$ Leiden, and antithrombin deficiency [5]. However, in certain cases, the etiology of thrombosis remains uncertain and this is when a possible pattern of platelet hyperaggregation as sticky platelet syndrome (SPS) could be considered as the cause of thrombosis [6].
The SPS was first described in the 80s as a thrombophilia in which qualitative alterations of the platelet function increase its aggregation capacity, favoring in this way thrombosis with described cases of cerebrovascular disease, acute myocardial infarction, and ischemic retinopathy [7]. Below, we present the case of a patient without history of thrombosis who developed a sudden dysfunction of the graft after living kidney transplantation and whose final diagnosis was SPS.

\section{Case Report}

We report the case of a 49 -year-old woman with $\mathrm{O} \mathrm{RhD}$ positive blood group and a family history of premature death of her father due to cerebrovascular disease. In her surgical history, she underwent transsphenoidal surgery for pituitary adenoma at the age of 40 , with replacement therapy with 


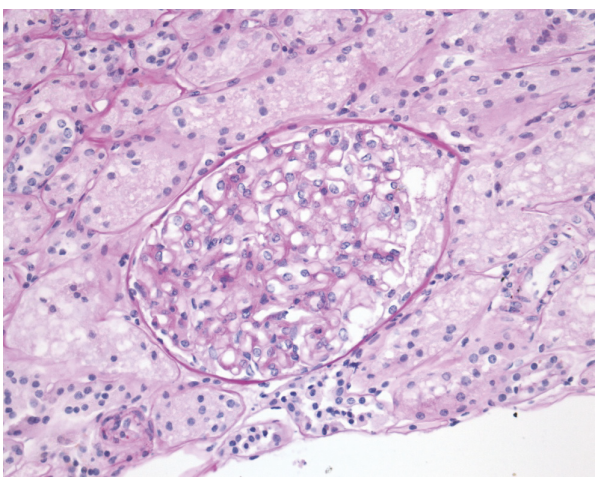

(a)

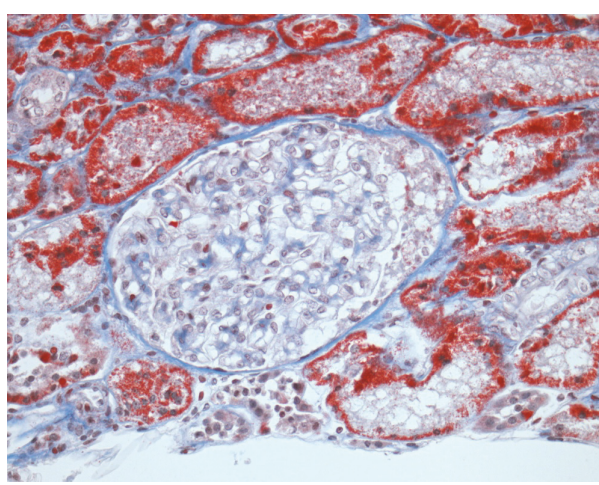

(c)

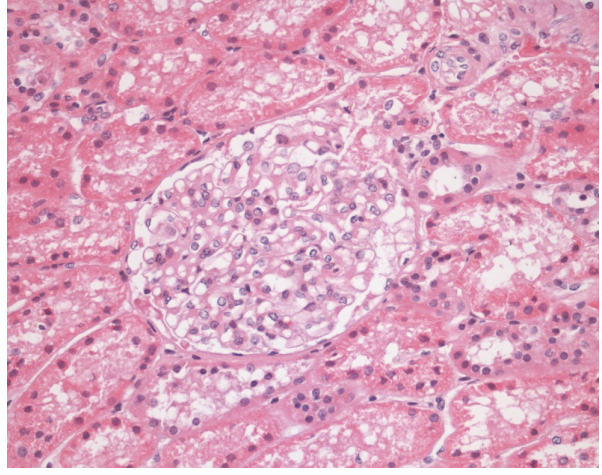

(b)

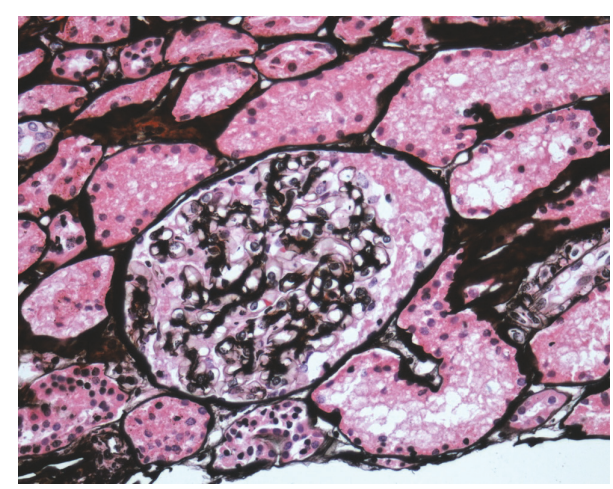

(d)

Figure 1: Zero-time biopsy of the renal graft. (a) Hematoxylin and eosin staining. (b) Periodic acid-Schiff staining. (c) Masson stain. (d) Silver methenamine stain. Renal tissue is visualized without significant vascular, tubular, and glomerular alterations. All photomicrographs are in 40x.

levothyroxine at a dose of $100 \mathrm{mcg}$ per day. The patient was diagnosed with type 2 diabetes mellitus at the age of 29 and has been treated since then with long-acting insulin. She also has a diagnosis of CKD in treatment with automated peritoneal dialysis from 47 years of age. A living-donor kidney transplant protocol was initiated, related to her sister (healthy 39-year-old woman), with whom she shares one haplotype and specific negative anti-donor antibodies.

The induction scheme was performed with basiliximab and methylprednisolone, with a cold ischemia time of 120 minutes. During surgery, arterial anastomosis was observed with progressive decrease of the thrill. For this reason, it was necessary to dismantle the anastomosis followed by exploration, observation, and discharge of a thrombus from the renal artery. The anastomosis was completed with adequate graft perfusion. The renal biopsy at time zero was without significant vascular, glomerular, and interstitial tubule alterations (Figures 1(a)-1(d)). After surgery, the patient remained in oligoanuria. A Doppler ultrasound of the graft was performed, which showed the renal artery throughout its course with high resistance pulsatile flow, with an average systolic flow velocity of $58.7 \mathrm{~cm} /$ second and inversion of the diastolic flow. The segmental, interlobar, and arcuate arteries in the upper, lower, and middle poles showed decreased systolic flow with an average of $34.1 \mathrm{~cm} /$ second. Meanwhile, the renal vein could not be identified at the time of the study (Figures 2(a) and 2(b)).

Ultrasound diagnosis was a thrombosis of the renal vein with hypoperfusion of the renal artery. The patient was admitted to the operating room and as a macroscopic finding the renal graft showed a purplish coloration and pallor, absence of thrill, complete anastomosis, and the presence of an intrarenal venous clot. Due to the damage, graft transplantectomy was performed and the graft was sent for revision by a pathologist who reported diffuse medullary cortical infarction and acute thrombotic microangiopathy with negative immunohistochemistry for C4d (Figures 3(a)-3(d)). The postsurgical follow-up was performed with the search for potential thrombophilias. The quantification of protein $\mathrm{C}$, protein $S$, and antithrombin was normal and the profile for anti-phospholipid antibodies was negative. In search of other pathologies, we requested studies of platelet aggregation, which showed platelet hyperaggregability at decreasing doses of epinephrine and normal aggregability associated with the exposure of adenosine diphosphate, compatible with sticky platelet syndrome type II (Figures 4(a) and 4(b)). The patient returned to automated peritoneal dialysis and management with acetylsalicylic acid as an antiplatelet agent was started. 


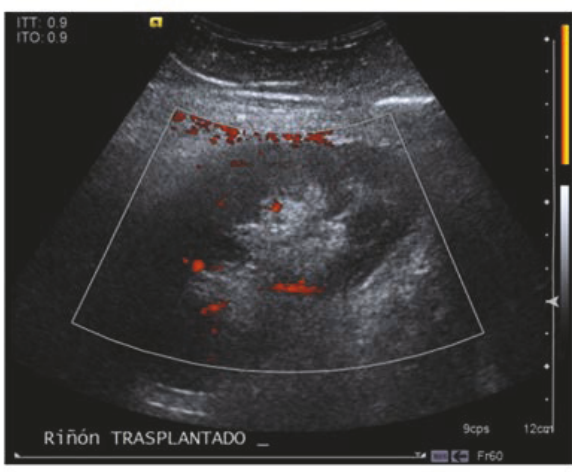

(a)

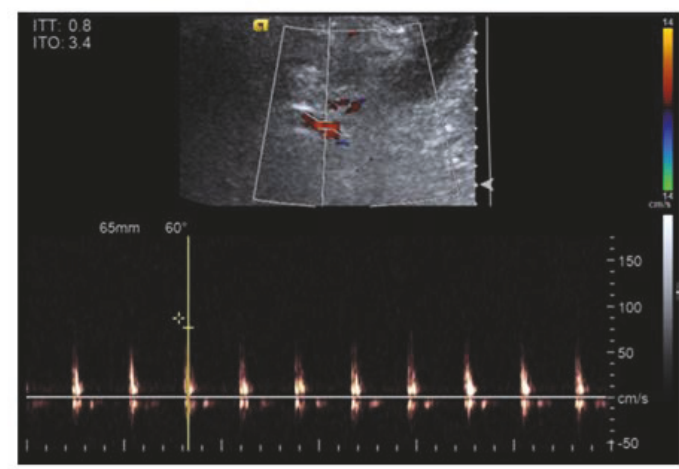

(b)

Figure 2: Doppler ultrasound. (a) Graft of the patient shows the absence of blood flow at the level of the renal cortex, arcuate arteries, interlobular arteries, and arterial anastomosis. (b) Duplex mode shows an arterial biphasic waveform with delayed acceleration, decreased systolic peak, and increased flow in diastole, which is a suggestive pattern of ischemia.

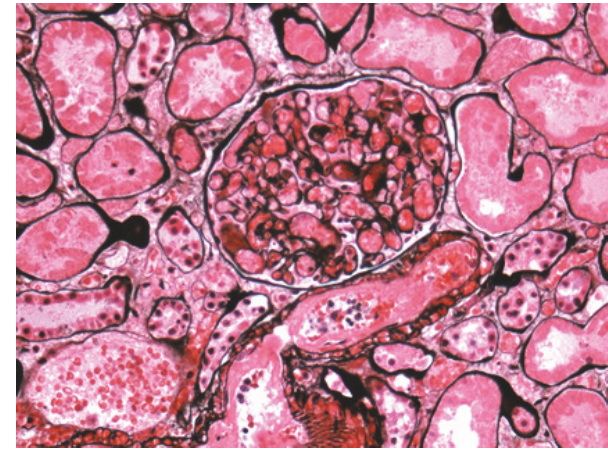

(a)

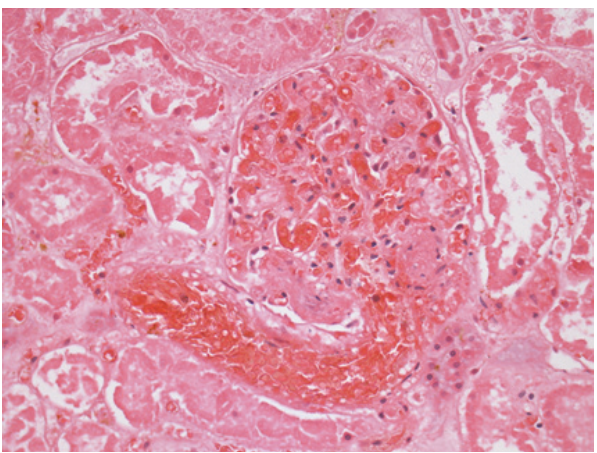

(c)

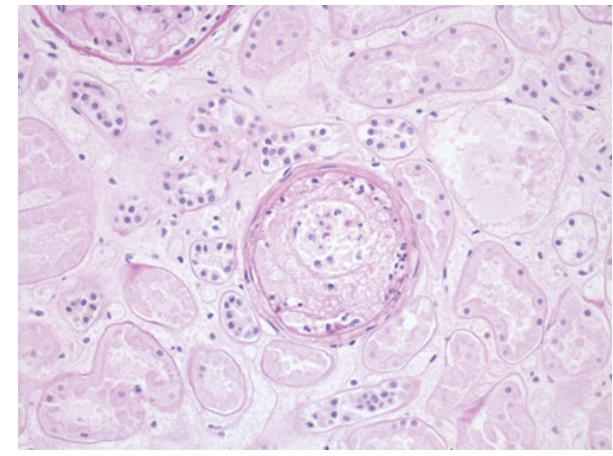

(b)

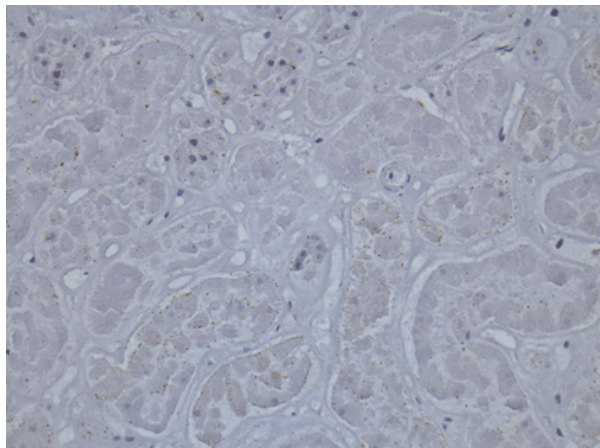

(d)

FIGURE 3: Micrographs of graft nephrectomy (40x). Stained, respectively, with Jones' Methenamine (a), PAS (b), Hematoxylin and Eosin (c), and indirect peroxidase immunostaining for $\mathrm{C} 4 \mathrm{~d}(\mathrm{~d})$. In all sections, fibrin thrombi adhered to the endothelium of the glomeruli ((a) and (c)) and the arteriolar walls (b) can be observed. C4d (d) was negative. All photomicrographs are in 40x.

\section{Discussion}

Described for the first time in the Ninth Stroke and Cerebral Circulation Conference by Holiday et al. [8], the sticky platelet syndrome is defined as a qualitative alteration of the platelet function and of autosomal dominant inheritance, characterized by platelet hyperaggregation in vitro with low concentrations of adenosine diphosphate (ADE) and/or epinephrine (EPI) but with normal aggregation in response to collagen, arachidonic acid, ristocetin, and thrombin [9].

Depending on the pattern of platelet aggregation, three different types of sticky platelet syndrome have been described. In type I, hyperaggregation is evidenced with both $\mathrm{ADE}$ and EPI. Type II shows hyperaggregation only with 


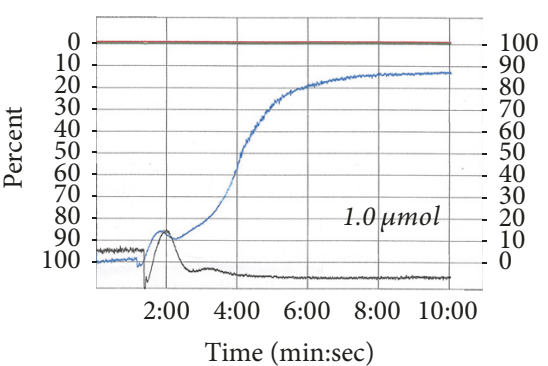

- Trace 1 Trace 3

- Trace 2 Trace 4

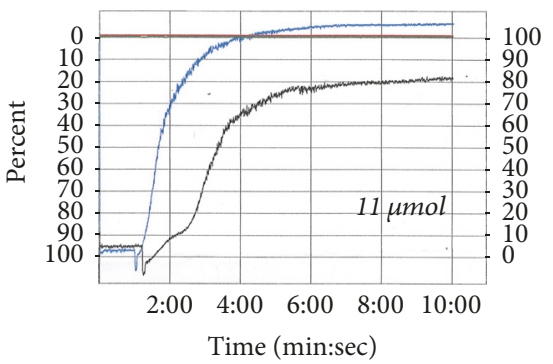

$\begin{array}{cr}\text { Trace } 1 & \text { Trace } 3 \\ \text { Trace } 2 & \text { Trace } 4\end{array}$

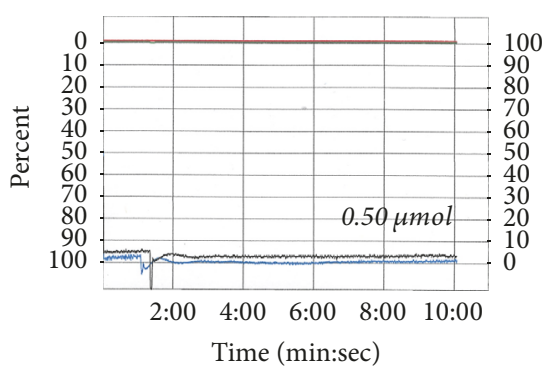

- Trace $1-$ Trace 3

— Trace 2 Trace 4

(a) Adenosine diphosphate (ADE)

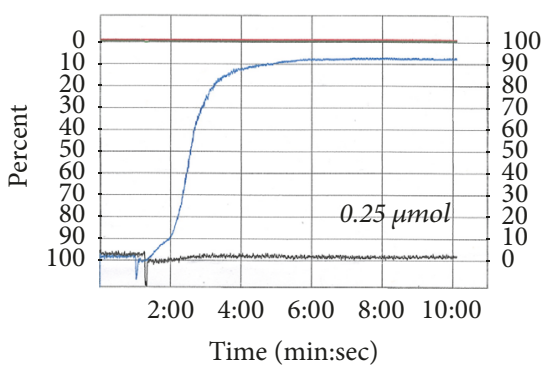

$\begin{array}{cr}\text { Trace } 1 & \text { Trace } 3 \\ \text { Trace } 2 & \text { Trace } 4\end{array}$

(b) Epinephrine (EPI)

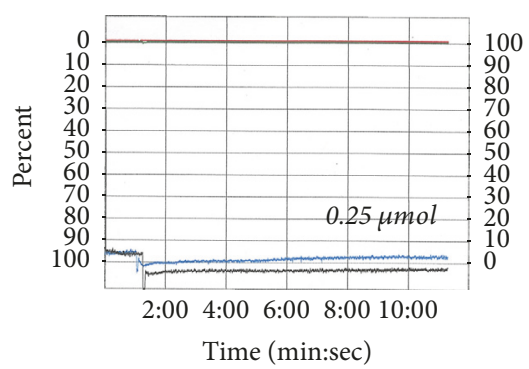

- Trace 1 Trace 3

- Trace 2 Trace 4

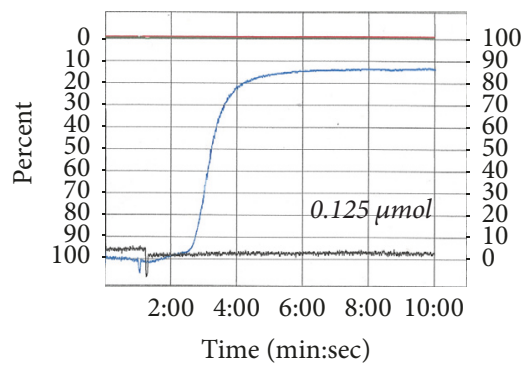

- Trace $1-$ Trace 3

FIGURE 4: Results of two platelet aggregometry tests: patient (blue line) and control (black line). (a) From left to right, the percentage of platelet aggregation with decreasing doses of ADE is shown. At $1 \mu \mathrm{mol}$, the patient presents platelet aggregation of almost $90 \%$ at $10 \mathrm{~min}$. At lower concentrations of ADE, the pattern of aggregation is similar to that of the control $(0.5$ and $0.25 \mu \mathrm{mol})$. (b) From left to right, the percentage of platelet aggregation with decreasing doses of EPI is shown. At $11 \mu \mathrm{mol}$, the patient and control present platelet aggregation that reaches more than $80 \%$ at $10 \mathrm{~min}$. In contrast to lower concentrations of EPI, while the control stops having platelet aggregation, the patient maintains a pattern of aggregation above $80 \%$ at concentrations of 0.25 and $0.125 \mu \mathrm{mol}$.

EPI, and in type III, hyperaggregation is seen only with ADE [10]. There are no specific epidemiological data on SPS, because the studies have taken place in very exclusive population groups and in patients with thrombosis without apparent cause. Prevalence has been reported in patients with thrombosis without apparent cause between 17.6 and $28 \%$ $[7,11]$, while in women with miscarriages it has been reported in $20 \%$ [12] and in $41 \%$ of patients on hemodialysis with recurrent thrombosis of vascular access [13].

Within the clinical picture presented by these patients, arterial thrombosis is the most frequent manifestation of the disease, followed by venous thrombosis [14]. A study conducted by the National Center of Haemostasis and Thrombosis of Slovakia characterized 360 patients with SPS, describing 233 patients $(64.7 \%)$ with arterial thrombosis and $127(35.2 \%)$ with venous thrombosis [14]. Other characteristic data of SPS are the presentation in young adults with no apparent risk factors, in people with a family history of thrombosis, in women with repeat miscarriages, and in patients who have thrombosis in unusual sites (retinal circulation, cerebral sinuses, etc.) and episodes of thrombosis which occur even in spite of adequate anticoagulation [9].

One of the laboratory tests that help evaluate platelet function is aggregometry, which measures the ability of some substances to induce in vitro platelet activation and aggregation.

The diagnosis of SPS is made through a study of platelet aggregation in which platelet overaggregation is demonstrated when exposed to ADE and/or EPI. The most widely used method is turbidimetry, which measures the average platelet aggregation from the difference in optical density between platelet-rich plasma (PRP) and platelet-poor plasma (PPP), when an agonist is added as ADP, epinephrine, collagen, and ristocetin, to name a few; as the platelets are added, they allow a greater passage of light, decreasing the optical density, yielding the result through the aggregation curves as a function of time. Another method of measurement used is impedance, which measures the increase in resistance to the passage of electrical current through 2 electrodes by placing whole blood in contact with an agonist when platelets begin to aggregate [15].

In 2007, Mühlfeld et al. reported three cases in which patients had presented thrombotic complications after a kidney transplant and were diagnosed with SPS after posttransplant thrombotic episodes. Mühlfeld proposed that the increase in adrenaline secretion due to preoperative stress was likely to induce the typically abnormal aggregation pattern of SPS [6]. However, there is a possibility that other 
mechanisms may be involved, such as alloimmune vascular damage, postoperative hypertensive episodes, and the use of immunomodulatory drugs such as calcineurin inhibitors [6]. From the description of SPS, Mammen proposed that the underlying cause was in alterations of membrane glycoproteins and their role in platelet activation [16]. Although there have been multiple studies, to date, it has not been possible to find a genetic alteration that explains this syndrome. Different membrane protein polymorphisms have been studied, such as mutations in GPIIIaPlA A1/A2 and in Gas6 c. $834+$ $7 \mathrm{G}>\mathrm{A}$, in which no statistically significant difference was found between the groups with SPS and controls [17, 18]. However, some polymorphisms of GP6 SNPs have been shown to be present more frequently in cases of SPS [19]. Despite these associations, there is still not enough evidence to define the etiology of SPS, which, due to its different forms of clinical presentation and inducibility characteristics of platelet aggregation, could have a multifactorial origin [9].

Because there are no treatment guidelines for SPS to date, the current recommendations are based on observations that acetylsalicylic acid (ASA) at low doses can normalize the pattern of platelet aggregation $[14,16]$. For this reason, it is recommended to start treatment with ASA at doses of 80 to $100 \mathrm{mg}$ every 24 hours [14]. However, for those patients who do not have an adequate response, it is recommended to scale the dose to $325 \mathrm{mg} /$ day. The use of ADP inhibitors such as clopidogrel could be recommended only if, despite the escalation of the ASA dose, there is no normalization of the platelet aggregation pattern or there is any contraindication to the use of ASA [20]. Although monitoring of the efficacy of platelet antiaggregants is not standardized, it is recommended that once the treatment with ASA or clopidogrel has begun, the aggregation tests should be reevaluated in order to achieve adequate efficacy.

A targeted search for SPS should be recommended if there is a history of thrombosis without a specific cause or when the patient on the transplant and hemodialysis waiting list has reports of recurrent thrombosis of vascular access, particularly in renal transplant recipients.

The preoperative treatment of a transplant recipient when they have a proven SPS is controversial. Traditionally, it is described that there is an increased risk of bleeding during a surgical procedure (approximately $20 \%$ only with aspirin and up to 50\% with aspirin and clopidogrel) [21]. However, there are studies in which it has been proven that the use of aspirin before and after transplantation reduces the risk of graft thrombosis without significantly increasing the risk of bleeding $[22,23]$. These studies use low doses of aspirin (75-150 mg), which seems to be a strategy to be used in patients with SPS who will undergo kidney transplantation [24].

In conclusion, SPS is a poorly recognized entity but with an adverse prognosis in patients with kidney transplantation. Its recognition must be done in the pretransplant period to avoid adverse complications in the postoperative period. Its diagnosis requires evidence of platelet aggregation and its treatment is relatively effective with the use of antiplatelet agents.

\section{Conflicts of Interest}

The authors declare that there are no conflicts of interest.

\section{References}

[1] N. Bakir, W. J. Sluiter, R. J. Ploeg, W. J. Van Son, and A. M. Tegzess, "Primary renal graft thrombosis," Nephrology Dialysis Transplantation, vol. 11, no. 1, pp. 140-147, 1996.

[2] A. O. Ojo, J. A. Hanson, R. A. Wolfe et al., "Dialysis modality and the risk of allograft thrombosis in adult renal transplant recipients," Kidney International, vol. 55, no. 5, pp. 1952-1960, 1999.

[3] J. T. Adler, J. F. Markmann, and H. Yeh, "Renal allograft thrombosis after living donor transplantation: risk factors and obstacles to retransplantation," Clinical Transplantation, vol. 30, no. 8, pp. 864-871, 2016.

[4] C. Ponticelli, M. Moia, and G. Montagnino, "Renal allograft thrombosis," Nephrology Dialysis Transplantation, vol. 24, no. 5, pp. 1388-1393, 2009.

[5] J. L. Kujovich, "Thrombophilia and thrombotic problems in renal transplant patients," Transplantation, vol. 77, no. 7, pp. 959-964, 2004.

[6] A. S. Mühlfeld, M. Ketteler, K. Schwamborn et al., "Sticky platelet syndrome: An underrecognized cause of graft dysfunction and thromboembolic complications in renal transplant recipients," American Journal of Transplantation, vol. 7, no. 7, pp. 1865-1868, 2007.

[7] R. L. Bick, "Sticky platelet syndrome: A common cause of unexplained arterial and venous thrombosis," Clinical and Applied Thrombosis/Hemostasis, vol. 4, no. 2, pp. 77-81, 1998.

[8] P. L. Holiday, E. Mammen, and J. Gilroy, "Sticky platelet syndrome and cerebral infarction in young adults," in Proceedings of the Ninth International Joint Conference on Stroke and Cerebral Circulation, Phoenix, Arizona, 1983.

[9] G. J. Ruiz-Delgado, Y. Cantero-Fortiz, M. A. Mendez-Huerta et al., "Primary thrombophilia in Mexico XII: Miscarriages are more frequent in people with sticky platelet syndrome," Turkish Journal of Hematology, vol. 34, no. 3, pp. 239-243, 2017.

[10] P. Kubisz, J. Stasko, and P. Holly, "Sticky platelet syndrome," Seminars in Thrombosis and Hemostasis, vol. 39, no. 6, pp. 674683, 2013.

[11] J. A. Andersen, Report: bleeding and thrombosis in women. Biomed Progress, 12: 40, 1999.

[12] R. L. Bick and D. Hoppensteadt, "Recurrent miscarriage syndrome and infertility due to blood coagulation protein/platelet defects: A review and update," Clinical and Applied Thrombosis/Hemostasis, vol. 11, no. 1, pp. 1-13, 2005.

[13] R. Klamroth, F. Seibt, and H. Rimpler, "Recurrent vascular access site thrombosis in patients on hemodialysis a problem of thrombophilia?” Blood, vol. 104, p. 300a, 2004.

[14] J. Sokol, M. Skerenova, Z. Jedinakova et al., "Progress in the Understanding of Sticky Platelet Syndrome," Seminars in Thrombosis and Hemostasis, vol. 43, no. 1, Article ID 02342, pp. 008-013, 2017.

[15] M. Martínez-Arias, B. López-Martínez, and I. Parra-Ortega, "Pruebas de laboratorio para la evaluación de la función de las plaquetas," Revista Latinoamericana de Patología Clínica y Medicina de Laboratorio, vol. 62, no. 4, pp. 245-252, 2015.

[16] E. F. Mammen, "Ten Years' Experience with the "Sticky Platelet Syndrome"," Clinical and Applied Thrombosis/Hemostasis, vol. 1, no. 1, pp. 66-72, 1995. 
[17] G. J. Ruiz-Argüelles, J. Garcés-Eisele, C. Camacho-Alarcón et al., "Primary thrombophilia in Mexico IX: The glycoprotein IIIa PL A1/A2 polymorphism is not associated with the sticky platelet syndrome phenotype," Clinical and Applied Thrombosis/Hemostasis, vol. 19, no. 6, pp. 689-692, 2013.

[18] P. Kubisz, L. Bartošová, J. Ivanková et al., "Is Gas6 protein associated with sticky platelet syndrome?" Clinical and Applied Thrombosis/Hemostasis, vol. 16, no. 6, pp. 701-704, 2010.

[19] D. Kotuličová, P. Chudý, M. Škereňová, J. Ivanková, M. Dobrotová, and P. Kubisz, "Variability of GP6 gene in patients with sticky platelet syndrome and deep venous thrombosis and/or pulmonary embolism," Blood Coagulation \& Fibrinolysis, vol. 23, no. 6, pp. 543-547, 2012.

[20] E. F. Mammen, "Sticky platelet syndrome," Seminars in Thrombosis and Hemostasis, vol. 25, no. 4, pp. 361-366, 1999.

[21] P. G. Chassot, C. Marcucci, A. Delabays, and D. R. Spahn, "Perioperative antiplatelet therapy," American Family Physician, vol. 82, no. 12, pp. 1484-1489, 2010.

[22] G. J. Murphy, R. Taha, D. C. Windmill, M. Metcalfe, and M. L. Nicholson, "Influence of aspirin on early allograft thrombosis and chronic allograft nephropathy following renal transplantation," British Journal of Surgery, vol. 88, no. 2, pp. 261-266, 2001.

[23] A. J. Robertson, V. Nargund, D. W. R. Gray, and P. J. Morris, "Low dose aspirin as prophylaxis against renal-vein thrompbosis in renal-transplant recipients," Nephrology Dialysis Transplantation, vol. 15, no. 11, pp. 1865-1868, 2000.

[24] J.-M. El-Amm, J. Andersenb, and S. A. Gruber, "Sticky platelet syndrome: A manageable risk factor for posttransplant thromboembolic events [3]," American Journal of Transplantation, vol. 8 , no. 2 , p. $465,2008$. 


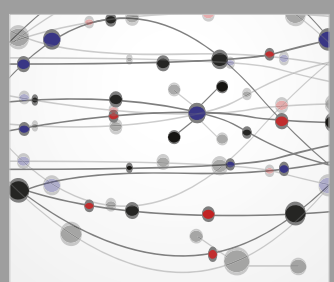

The Scientific World Journal
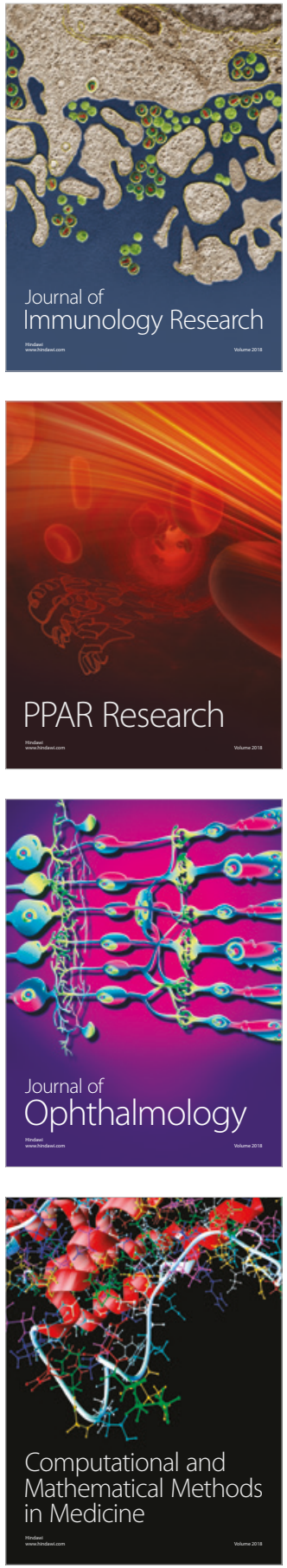

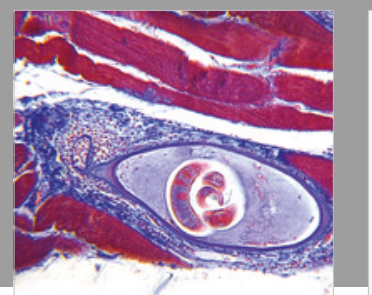

Gastroenterology Research and Practice

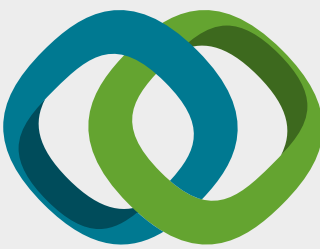

\section{Hindawi}

Submit your manuscripts at

www.hindawi.com
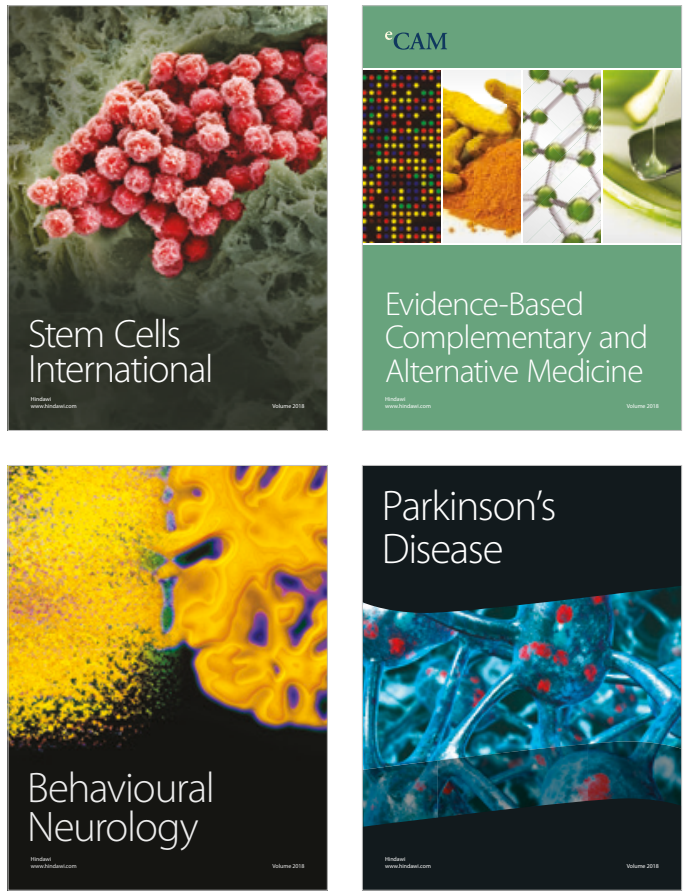

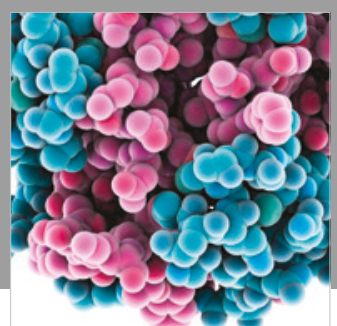

ournal of

Diabetes Research

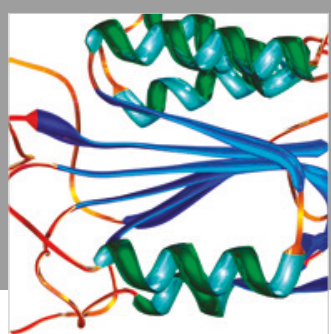

Disease Markers
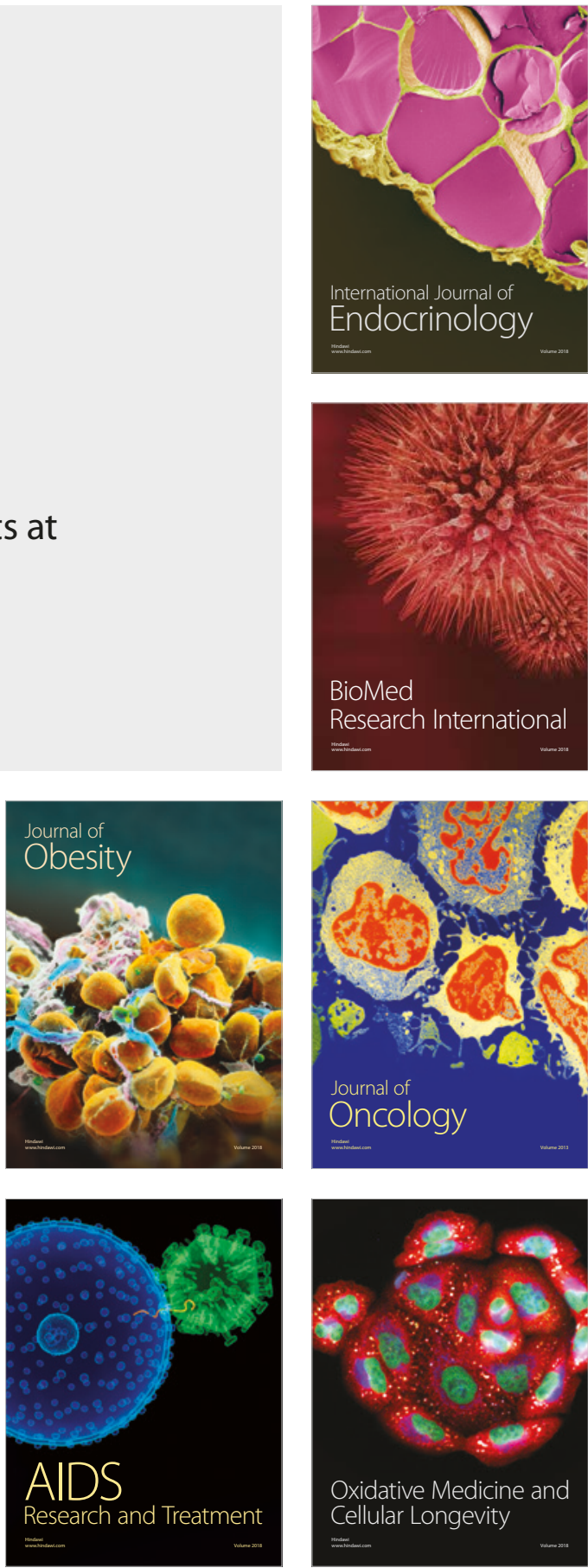